Horizons philosophiques

\title{
Compte rendu d'une table ronde sur Curiosités esthétiques
}

\section{Mona Hakim}

Volume 3, numéro 2, printemps 1993

Paysages esthétiques

URI : https://id.erudit.org/iderudit/800926ar

DOI : https://doi.org/10.7202/800926ar

Aller au sommaire du numéro

Éditeur(s)

Collège Édouard-Montpetit

ISSN

1181-9227 (imprimé)

1920-2954 (numérique)

Découvrir la revue

Citer ce compte rendu

Hakim, M. (1993). Compte rendu de [Compte rendu d'une table ronde sur Curiosités esthétiques]. Horizons philosophiques, 3(2), 115-123.

https://doi.org/10.7202/800926ar d'utilisation que vous pouvez consulter en ligne.

https://apropos.erudit.org/fr/usagers/politique-dutilisation/ 


\section{COMPTES RENDUS}

Compte rendu d'une table ronde sur Curiosités esthétiques

L'exposition Curiosités esthétiques (titre tiré d'un essai de Baudelaire, paru en 1860) s'est tenue simultanément dans les huit lieux de diffusion attitrés de la Montérégie en décembre dernier, chaque institution présentant un groupe d'artistes distincts. Organisé par le Regroupement des diffuseurs en arts visuels de la Montérégie (RDAVM), cet événementcollectiffutconçudansle butde contrerlabanalité des objetsusuels : des objets qui, assujettis aux symboles de la mode et identifiés à un produit de masse, perdent à nos yeux tout pouvoir d'évocation.

C'est dans cette perspective que les artistes ont créé à partir d'objets dits fonctionnels des oeuvres ingénieuses, insolites et/ou loufoques, stimulant ainsi notre sens de l'étonnement. Or, entre l'objet d'art et une relecture de nos objets quotidiens, où commence la curiosité? Toute oeuvre d'art n'est-elle pas curiosité esthétique? Ces interrogations constituaient le thème de la table ronde organisée par la Galerie d'art du Collège Édouard-Montpetit conjointement à l'événement.

Pour débattre le sujet, la table ronde réunissait :

GHYSLAINE GUERTIN, professeure de philosophie au Collège Édouard-Montpetit et membre de la Société canadienne d'esthétique; JEAN-PIERRE LEGRAND, critique d'art; LOUISE POISSANT, professeure à la maîtrise en arts plastiques à P'UQAM et membre de la Société d'esthétique du Québec; NORMAND THÉRIAULT, conservateur et critique d'art; MICHEL GROLEAU, directeur du centre d'exposition Expression à Saint-Hyacinthe, membre du RDAVM et MICHELLE HÉON, artiste et participante à Curiosités esthétiques; MONA HAKIM, critique ethistorienne de l'art, agissaità titre de modératrice.

Nous publions ici le compte rendu des énoncés de cette table ronde rédigéà la suite d'une transcription des enregistrements. Le document sonore se prêtant moins à la précision que le document écrit, notre texte doit être considéré comme une interprétation en résumé des concepts élaborés par les six intervenants. Les traductions qui suivent sont présentées par ordre d'intervention.

Pour GHYSLAINE GUERTIN, la question proposée pour ce débat concerne d'emblée le pôle de la réception de l'oeuvre d'art en rapport avec l'attitude, le comportement, la réaction de celui ou celle à qui elle s'adresse. En effet, lorsque 
Baudelaire affirme que le pouvoir de l'artconsisteà susciter l'étonnement, il s'empresse d'ajouter : "C'est un bonheur d'être étonné. /C'est un bonheur de rêver'." Le sens du concept d'étonnement, au centre même de ses propos sur le beau, le bizarre, est également directement lié à celui de l'admiration et du plaisir.

Toutefois, poursuit Ghyslaine Guertin, si l'on veut approfondir le sens de l'expérience esthétique, il importe de montrer les rapports qu'entretiennent le pôle de la réception avec ceux de la production de l'oeuvre et de l'oeuvre elle-même. Baudelaire nous invite à effectuer cette démarche lorsqu'il se penche sur les conditions de l'étonnement et la relation entre l'étonnement et le beau. II se tourne vers l'oeuvre d'art en montrant qu'elle doit échapper aux règles et comporter un aspect de nouveauté. Le beau ne saurait être banal. II contiendrait toujours, selon Baudelaire, "une dose de bizarrerie" ${ }^{2}$, qui le fait être étonnant. Par ailleurs, "suffirait-il à un objet d'être étonnant pour être beau?" se demande la philosophe, en reprenantl'interprétation de Baudelaire.

Cette question renvoie Baudelaire au pôle de la production de l'oeuvre. II s'en prend auxartistes qui veulentà toutprix surprendre, étonner, plaire au public en se conformant à son goût par les voies de la facilité et par des "moyens étrangers à l'art" L'imagination de l'artiste, au delà de tout conformisme, doit être au centre même du processus créateur conduisantà l'expression de l'individualité et de la beauté. Dans ce cas, l'objet le plus simple s'offrant à la perception est susceptible de déclencher l'étonnement et de conduire à un processus de réflexion et de questionnement, comme l'a si bien montré Paul Valéry dans son texte L'Homme et la coquille', note Ghyslaine Guertin. Tout au long de sa recherche, Valéry nous présente l'étonnement non seulement comme le principe moteur de la connaissance mais aussi du plaisir esthétique.

Cette notion de plaisir fait partie intégrante de l'expérience esthétique. Elle a été malheureusement méconnue par toute la tradition spéculative de l'art, souligne G. Guertin. Il semble nécessaire de l'intégrer dans la réflexion actuelle et de montrer, à l'instar de Jean-Marie Schaeffer, qu' 'elle est inséparable d'une attitude cognitive (perceptive et conceptuelle) et donc aussi d'une attention soutenue à ce que l'oeuvre d'art peut nous apprendre ${ }^{\text {"I }}$. II s'agit là d'une démarche qui s'applique non seulement au domaine des arts visuels mais également à celui de l'art littéraire et musical.

1. Charles Beaudelaire, Curiosités esthétiques, Paris, Calman-Lévy, 1860, p. 258.

2. Ibid., p. 217.

3. Ibid., p. 258.

4. Paris, Gallimard, «Bibliothèque de la Pléiade», 1957, p. 886-907.

5. Jean-Marie Schaeffer, L'Art de l'áge moderne, Paris, Gallimard, p. 386. 
JEAN-PIERRELEGRAND a procédéà une définition de la curiositéà partir de mises en situation des individus. Chez lui, la curiosité s'assimile à un désir de connaissance, celui-ci répondant expressémentà un besoin de survie. En somme, l'humain sonde son environnement à la recherche de tout ce qui menacerait ou améliorerait ses chances de survie. Reposant ainsi surune déstabilisation, la curiosité tire profit d'une impression que quelque chose dans le monde nous échappe (survie menacée), ou sinon elle provoque un sentiment de découverte qui nous oblige à repenser notre place dans le monde (amélioration de nos chances de survie).

Jean-Pierre Legrand compare la curiositéa la vision d'un enfant toujours prêtà jeter un regard neuf sur ce qui l'entoure. Aux yeux de l'enfant, tout est toujours nouveau. Un système qui se replie sur lui-même, soit un système imperméable à linformation nouvelle, provient d'une prétention à tout connaître et à croire que toute nouveauté ne changera rien à notre condition. Toujours selon lui, ce refus d'assimilation à de nouvelles données répondàl'absence de curiosité de plus en plus manifeste aujourd'hui. Sur un autre plan, la curiosité esthétique, comparableà la survie de l'esprit, correspond judicieusement au travail de l'art. Un travail propice à stimuler de nouvelles découvertes, comme le font fort bien les objets présentés dans ce projet d'exposition, souligne le critique d'art.

Une sorte de révolution intellectuelle se produit au moment où nous dressons une cartographie intérieure incluant une période de crise lorsque nous sommes obligés de repenser cette carte de la réalité. J.-P. Legrand réfère ici aux cartes intérieures prélevées d'un essai de Scott Peck. Nous faisons rapidement le tour d'un territoire que nous avons dans un premier temps exploré et par la suite balisé, car tout notre entourage nous encourage à tenir notre territoire comme acquis pour aussitôtl'oublier. Orles découvertes nouvelles obligent précisémentà une constante réévaluation. Et du reste, la vie, avec ses épreuves et ses paradoxes, se charge d'éveiller notre mémoire en nous incitant à chercher à nouveau, ne serait-ce que pour parvenir à trouver des solutions à nos problèmes. Les artistes, précise Jean-Pierre Legrand, sont les porteparole tout indiqués pour nous remettre en position d'étonnement et nous permettre de revoir le monde un peu à la manière du regard des enfants.

Le discours de LOUISE POISSANT porte davantage attention àl'objet (matériel) de curiosité. Elle relate ainsi la transformation de l'objet en fétiche tel que pratiqué par les Cabinets de curiosités au XVI ${ }^{\ominus}$ siècle, jusqu'au déclin de son règne engendré par la société actuelle. 
Aux siècles derniers, les antiquités de toute sorte furent considérées dans un esprit de curiosité et dans un but quantitatif, plutôt que pour leur valeur d'usage. Or avec les Modernes du XIX' siècle, la curiosité se voit de plus en plus associée à la nouveauté; à l'inverse de la notion de conservation, la nouveauté suggère un remplacement rapide de l'objet, soutient Louise Poissant. À l'ère contemporaine, l'avènement de la télévision a orienté le public vers une banalisation de l'objet provoquant ainsi l'apparition d'une nouvelle esthétique. Parce qu'elle propose des contenus exotiques et étrangers, corroborés par une culture multiethnique, plus rien à nos yeux ne devient étonnant ou bizarre. La profusion d'images projetées à un rythme effréné rend littéralement impossible une ingestion intégrale, poursuit-elle. Le direct rend le phénomène encore plus troublant car ce ne sont plus des objets que l'on documente mais bien des événements chargés d'une dimension spectaculaire. Ceux-ci auront à plus ou moins long terme éliminé l'emphase ou la fétichisation de l'objet tout court.

En ce qui concerne cette dématérialisation de l'objet, Louise Poissant souligne l'apport des artistes impliqués dans l'univers technologique. Travaillant sur les enjeux d'une technologie qui habite notre quotidien, souven tà notre insu, ces artistes scrutent, questionnent, démontent et reconstruisent l'univers, le tournent en dérision ou encore le réinventent et le domestiquent. Leurs oeuvres ne se résument pas à un bricolage high-tech mais exploitent l'accès des dispositifs de leurs constructions. Ils s'agit pour eux de faire voir dans quelle direction se transforme notre environnement. Mais à long terme, la disparition ou la dématérialisation de l'objet se fera, entre autres, au profit d'une intensification des relations humaines et d'une spiritualisation des engagements, affirme-t-elle. Des médiations technologiques seront favorisées parles cyberspaces ou la réalité virtuelle. On note déjà un accroissement du rôle actif des partenaires par une multiplication des dispositifs interactifs.

Louise Poissant relève icila production de certains artistes de l'exposition Curiosités esthétiques qui ont semblé le plus correspondre aux tendances mentionnées plus haut. II s'agit d'oeuvres qui jouent à leur façon avec la technologie, sans qu'il soit toutefois question d'électronique. Elle mentionne, entre autres I'oeuvre dans laquelle se fontface deuxventilateurs (Cyrille Levi), où le bruitdel'uns'annihile par rapportàl'autre, simulant un dialogue de sourds. L'artiste dénonce ainsi la pauvreté des échanges communicationnels actuels en mettantl'accent surleurs dispositifs factuels. Ailleurs, un autoportrait transcrit sur un film radiographique (Louise Paillé) projette une vue à la fois la plus intime et la plus anonyme de l'identité. Un autre artiste a pratiqué des autopsies sur des objets tels que le pacemaker ou le masque à gaz, par le biais de coupe latérale (Daniel-Jean Primeau). Ces coupes révèlent à la fois la structure et une partie du 


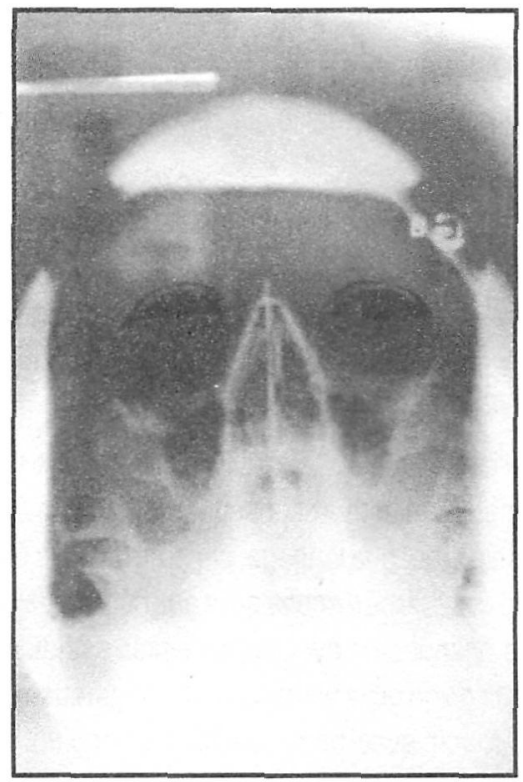

Louise Paillé, Autoportrait aux yeux, 1992

fonctionnement de l'objet tranché. Selon L. Poissant, les objets de cet artiste parodient les Cabinets de curiosités du fait qu'ils ne sont pas, comme les objets de certains d'entre eux, basés sur le seul point de vue de l'analogie des formes.

Louise Poissant conclut par un questionnement, demandant ce qu'il en est de l'objet esthétique compte tenu d'une réalité virtuelle imminente. Àson avis, nous n'aurions pas à craindre, malgré tout, sa disparition.

Selon NORMAND THÉRIAULT, une curiosité tientà ce qu'un objet créera suffisamment d'étonnement pour en parler. Ce pourrait être l'oeuvre complète de Barnett Newman, de Delacroix ou de Manet, présentée au même moment dans un même musée, donne à titre d'exemples l'historien d'art.

Sceptique par rapport à son engagement dans ce débat, Normand Thériault souligne les dangers inhérents aux curiosités esthétiques. Dans un premier temps, le concept peut être réducteur, de la même manière que le sort réservé à l'art par les Cabinets de curiosités : un art d'isolement, possédant de moins en moins d'importance. $N$. Thériault évoque ici la notion particulière d'éloignement telle que prônée par Baudelaire : le monde n'ayant plus de signification ni d'intérêt, il revient à l'art de lui 
donner un sens. Ainsi faut-il s'éloigner de tout ce qui est la réalité. Cette conception induira plus tardle conceptde l'art pourl'artavec tous les débats que l'on connaît. Selon I'historien d'art, c'est ce rapportà la réalité qui, dans les curiosités esthétiques, se veut dérangeant en soi.

Normand Thériault prendra à son compte les deux théories distinctes de Platon, à savoir que, d'une part, l'art est magique, qu'il consiste en une folie, en un désir qui ravit etque, d'autre partdans Les Lois, il n'est finalementqu'un divertissementinoffensif. Pris dans sa globalité, le système de Platon ferait de l'art un représentant de la réalité, alors que la réalité elle-même représente imparfaitement l'idée.

Du point de vue de l'esthétique $\mathrm{N}$. Thériault voit là un autre problème. L'esthétique le ramène aux catégories d'Aristote et conséquemment à l'évincement de l'art. À ce stade, l'esthétique, prise en tant que langage, servirait de grille établie devant la réalité. D'où le danger de l'usage exacerbé de cette grille aux dépens de ce qui se situe derrière. II se produirait le même phénomène avec les curiosités esthétiques. Tout discours sur ce thème peuts'assujettir à une grille extrêmement dangereuse en démontrant que l'art a de moins en moins à voir avec notre réalité. Alors qu'en parlant des Curiosités esthétiques, Baudelaire tentait de rejoindre une réalité qui était celle de l'artiste, aujourd'hui, poursuit l'historien d'art, on essaie d'éclipser tout ce qui est artistique pour laisser place au percepteur. Du côté de la critiqueactuelle, lagrille devientmanifestement plus lourde que l'art, que ce soit par l'effet de la phénoménologie ou par l'effet d'autres modèles d'analyse.

En guise de conclusion, Normand Thériaultse demande commentune création peut être une curiosité utile. L'art se situe-t-ill, comme chez Platon, au niveau de la folie et du délire, ou plutôt au niveau du divertissement inoffensif? Le danger avec les curiosités esthétiques, toujours selon lui, serait en fait dépendant du sens que l'on retient.

MICHELLE HÉON parle, quant à elle, de son expérience personnelle en tant qu'artiste dans cette aventure des Curiosités esthétiques. Elle évoque la hantise provoquée par ces deux mots. Parmi ses nombreux questionnements, elle relève : Estce que ma recherche est assez près des curiosités esthétiques? De quel point de vue un objet est-il curieux? Du point de vue de son auteur? Du point de vue du spectateur ou de celui du public élargi? Jusqu'à quel point dois-je faire une concession dans ma recherche actuelle pour donner place à une curiosité; suis-jeà l'aise dans ce contexte?

Les objets de cette artiste ne possèdent pas de fonction réelle évidente. Selon ses dires, les objets réels n'ont pas de véritables incidences dans sa production. Le concept ou le symbole liéa à la transformation de ses objets transcende leur simple représenta- 
tion. La curiosité esthétique passerait donc par la transformation de l'objet usuel et positionnerait ce dernier, ainsi que l'artiste, dans le temps et comme reflets d'une société.

Michelle Héon nous met en présence d'un exemplaire des Curiosités esthétiques de Baudelaire édité en 1917 par M. Auguste Poulet-Malassis. Un volume qui lui a été d'un grand réconfort, tient-elle à préciser, tant par le format et la couleur de l'objet que par son contenu. L'artiste dans son essai de 1860 cite ici Baudelaire : "La variété est une condition sine qua non de la vie. L'étonnement [...] tientà cette variété même des types et des sensations. [...] le beau est toujours bizarre. [...] Tâchez de concevoir un beau banal.

L'artiste conclut que, dans un contexte où les modes rendent les choses uniformes et banales et leurs spectateurs souvent blasés, des défis comme ceux posés par le projetdes Curiosités esthétiquesse veulentsalutaires pour les artistes; ne serait-ce que pour la réflexion portée sur le sujet ou sur notre mode de fonctionnement créateur.

Selon MICHEL GROLEAU, la curiosité estavant toutune question d'attitude. À partir du moment où nous nous investissons dans cette voie, s'ensuit une remise en question de nos jugements coutumiers. Àce titre, la curiosité peuts'interpréter indépendamment de la notion d'esthétique. Les critères d'esthétique, en cette fin de siècle, demeurent relatifs à chaque groupe d'individus, poursuit-il. L'importance réside davantage dans l'évocation d'un signe projeté par l'objet que d'aucuns perçoivent ou admettent encore difficilement. Seul le processus de décontextualisation et de recontextualisation d'un objet sera porteur d'une signification autre.

Michel Groleau évoque le problème de cette fin de siècle où se succèdent à un rythme affolant les mouvements artistiques. Ce faisant, l'art s'immisce dans le marché du bien de consommation. Un marché qui dicte le goût du jour, un art régi par les phénomènes de mode, dans lesquels tous peuvent se retrouver. En cela l'esthétique suit une règle qui évacue toute perspective d'étonnement.

Le galériste rappelle que le projet collectif de Curiosités esthétiquesse voulait avant tout une occasion d'étonner le public tou tautant que les organisateurs de l'événement. Le projet devaitêtre pris avec un brin d'humour sans être restreintà un discours par trop sérieux. L'objectif a été atteint, souligne-t-il. Certains ont proposé des réflexions surnos habitudes de vie, d'autres sur nos mécanismes sociaux, d'autres encore sur nos perceptions, toujours en respectant, en outre, leurs préoccupations premières. À ses yeux, nous devons surtout retenir l'énorme générosité de leur part, tenant lieu de facteur commun dans chacune des expositions. 


\section{Le public}

À la suite des commentaires des six participants, les interventions percutantes du public ont su aiguiser le débat. NORMAND THÉRIAULT fait d'abord état de la tendance actuelle en art fortement axée sur l'éducation. L'intérêt devient de moins en moins marqué pour les débats de fond, favorisant un climat propre à évacuer tout contenu en art. Nous sommes plus préoccupés à enseigner des solutions qu'à entretenir des questionnements; par ailleurs, la place est donnée aux événements et ce, aux dépens de la densité du discours, tient-il à préciser.

Ces constats trouvent leur écho parmi le public. Une intervenante signale le manque de nouveauté dans la production actuelle. Les artistes savent bien travailler, remplissent les critères d'excellence dans leur présentation de dossiers mais ne se questionnent pas. Ils empruntentn'importe quoi et semblent de plus en plus régresser. Le milieu de l'art serait plus vivant si les écoles formaient davantage de penseurs, opine-t-elle. L'artiste est porteur d'une réflexion qui doit rejoindre l'universel.

Une autre intervenante s'interroge sur la notion d'esthétique à partir de la trajectoire qu'a subie celle-ciau fil des siècles. Tandis qu'avec la mort de la rhétorique estapparue la théorie des symboles de Todorov, et qu'une vision de l'utile chez les Romantiques a cédé le pas au pouvoir de l'inutile et à des notions d'art pour l'art, qu'en est-il de l'esthétique actuelle après la déclaration de la mort de l'art? À son avis, nous sommes revenus à un art politique, propagandiste, éducatif, mais surtout pas inutile. Cette période d'esthétique exacerbée ne serait-elle la conséquence de notre académisme, lequel formerait une homogénéisation des productions, se demande cette même intervenante? L'artiste serait formé à partir de règles implicites dont les conséquences standardiseraient les procédés de production et de réception. Nous sommes loin, affirme-t-elle à l'instar de Normand Thériault, d'une période où l'étendard concernaitle fait de l'art, d'une période pendant laquelle les objets en tant que tels n'avaient aucune importance.

LOUISE POISSANT signale son désaccord avec ce type de réflexion. À son avis, les expositions diffèrent suffisamment entre elles et les multiples formes d'art jouent fort bien chacune les miroirs de notre société. L'art ne concerne pas que des artistes, souligne-t-elle, mais concerne également l'enseignement, le public, etc. Certes, il est sujet de ce fait à une certaine banalisation, mais il est plus ou moins important d'en tenir rigueur. Les étudiants en place sensibiliseront d'autres gens et créeront ainsi un rayonnement. Le développement progressif chez le public d'une sensibilité autre à l'égard d'une catégorie d'objets, de lieux ou de contacts doit être davantage à considérer. Louise Poissant fait par ailleurs remarquer que la relation qu'entretient 
l'esthétique avec l'objet est avant tout basée sur la sensibilité. En raison de la mort de la métorique, nous entrons bien sûr dans une esthétique généralisée. Les considérations portent dorénavant sur la façon d'esthétiser nos relations, conclut-elle.

Pour sa part, GHYSLAINE GUERTIN réitère icil'orientation de plus en plus marquée vers le pôle de la réception. Une tendance qui a suppléé aux théories spéculatives, incluant la notion de l'art pour l'art, apparue avec Kant dès le début du XIX ${ }^{\ominus}$ siècle. C'est ce qui fait croire à Ghyslaine Guertin en la nécessité de créer l'événement, que ce soit en art ou en musique contemporaine, là où, dans les deux cas, l'adhésion se fait plus difficilement.

Par sa double expérience comme artiste et enseignante, MICHELLE HÉON perçoit bien un danger venant de la part d'étudiants plus habiles à utiliser des formules : ils écrasent d'autres excellents artistes qui ne possèdent pas assez de culot pour lutter contre un système trop lourd.

Voilà proposées deux définitions de ces Curiosités esthétiques, tributaires ici de préoccupations centrées sur le pôle de la réception chez les uns, et sur le statut formel de l'objet chez les autres. Or, pour différentes qu'elles soient, ces deux méthodes d'appréhension de l'objet d'art n'échappent pas à des problématiques de base (qui ne sont donc pas nouvelles en elles-mêmes) concernantla "qualité", voire l'homogénéité des pratiques artistiques, qu'elles soient attribuables ou non à notre système d'éducation universitaire. Bien qu'ici ces problématiques débordaient le cadre théorique initial, elles révélaient somme toute des malaises trop souvent esquivés mais toujours latents dans le milieu des arts visuels. En outre, elles ne sont pas sans permettre une réévaluation (salutaire) des critères esthétiques actuels.

Mona Hakim

Galerie d'art du Collège Édouard-Montpetit 